\title{
Noninvasive pacing during transfemoral implantation of the Edwards SAPIEN valve for tricuspid valve bioprosthesis stenosis
}

\author{
Marcin Demkow ${ }^{1}$, Witold Rużyłło ${ }^{1}$, Sebastian Bujak ${ }^{1}$, Marek Konka², Piotr Szatkowski ${ }^{3}$, Barbara Lubiszewska ${ }^{1}$ \\ ${ }^{1}$ Department of Coronary and Structural Heart Diseases, Institute of Cardiology, Warsaw, Poland \\ ${ }^{2}$ Department of Congenital Heart Diseases, Institute of Cardiology, Warsaw, Poland \\ ${ }^{3}$ Department of Anesthesiology, Institute of Cardiology, Warsaw, Poland
}

Adv Interv Cardiol 2016; 12, 1 (43): 57-60 DOI: $10.5114 /$ pwki.2016.56951

\section{Introduction}

Transcatheter implantation of balloon expandable valves in high-risk patients with degenerated tricuspid bioprostheses has been reported since 2010 [1, 2]. Although there have been cases where the SAPIEN valve was implanted in the tricuspid position without rapid pacing $[3,4]$, invasive rapid ventricular pacing is usually applied during valve deployment.

We present a case report documenting for the first time the use of noninvasive, external rapid pacing delivered through defibrillation sticker-pads during transfemoral implantation of the Edwards SAPIEN XT valve for tricuspid valve bioprosthesis stenosis.

\section{Case report}

A 76-year-old woman with a history of rheumatic fever was treated at the age of 21 with open mitral commissurotomy. In 1995, at the age of 57 she underwent simultaneous triple valve replacement with mechanical aortic (21-mm Sorin Bicarbon; Sorin Biomedica, Saluggia, Italy), mitral (27-mm St. Jude medical valve; St. Jude Medical Inc., St. Paul, MN) prostheses and a tricuspid bioprosthesis (TBP) (29-mm Intact Valve; Medtronic Inc, Minneapolis, MN, USA). For the last 2 years she had experienced a decline in exercise tolerance and peripheral edema. At the beginning of 2015 she presented to our department with the New York Heart Association (NYHA) functional class III/IV and right heart failure. Despite aggressive treatment she continued to deteriorate. Her other medical history included paroxysmal atrial fibrillation, hypertension, chronic obstructive pulmonary disease, previous surgery for brain menin- gioma and right sided mastectomy for breast cancer. Echocardiography showed severe TBP stenosis with trace regurgitation and normal function of both mechanical valves. The mean and peak tricuspid gradient was $22 \mathrm{~mm} \mathrm{Hg}$ and $34 \mathrm{~mm} \mathrm{Hg}$ respectively. The left ventricle was not dilated, with preserved systolic function. The inner TBP diameter was measured at 21-22 mm by computed tomography and 20-21 mm by echocardiography. The heart team agreed that operative risk was prohibitive (calculated EuroSCORE II and logistic EurOSCORE were respectively $7.99 \%$ and $22.76 \%$ ) and transvenous tricuspid valve-in-valve implantation would be a possible life-saving option.

\section{Procedure}

The procedure was carried out in general anesthesia and with 3D transesophageal echocardiography (3D TEE) guidance. Right femoral venous access was used. The TBP was crossed with a 6 Fr multipurpose catheter placed within a Transseptal Guiding Introducer (8 Fr, $4.7 \mathrm{~cm}, 180^{\circ}$ curved tip, St Jude Medical Minnetonka, MN, USA). This coaxial system enabled the valve to be crossed and the right lower pulmonary branch artery to be cannulated easily (even with a severely enlarged right atrium). Predilatation of the TBP was performed with the $20 \mathrm{~mm} / 4 \mathrm{~cm}$ Mullins $X$ balloon (NuMED, Hopkinton, NY, USA) introduced over a 0.035-inch Amplatz Extra-Stiff guidewire (Cook Medical Inc, Bloomington, IN, USA) "anchored" in the right lower lobe pulmonary artery. No rapid pacing was applied. The 26-mm SAPIEN XT Valve was mounted over the NovaFlex+ (Edwards Lifesciences, Irvine, CA, USA) delivery system in the reverse 
orientation to transfemoral transcatheter aortic heart valve (TAVI). The valve was then delivered over a 0.035 inch Amplatz Extra-Stiff guidewire (Cook Medical Inc, Bloomington, IN, USA). The SAPIEN XT valve was placed in front of the mouth of the TBP, adjusting its position with the NovaFlex catheter flexion. By pushing the system forward with the pusher in place, the SAPIEN XT was precisely positioned in the TBP (Figure $1 \mathrm{~A}$ ). The valve was very slowly deployed under rapid pacing at $140 \mathrm{bpm}$ with a perfect final position and no residual leak (Figures $1 \mathrm{~B}, \mathrm{C}$ ). Noninvasive, external pacing (Philips HeartStart MRx ALS Defibrillator) through defibrillation stick- er-pads (Adult Multi-Function Defibrillation Electrodes, Medi-Trace TM Cadence, COVIDIEN TR) was applied. The electrodes were positioned on the left and right posterior axillary line. The current pulse was gradually increased from 50 to $175 \mathrm{~mA}$ until capture was noted. The directly measured maximal and average transtricuspid gradient decreased from 12 to 7 and from 9 to $4 \mathrm{~mm} \mathrm{Hg}$ respectively (Figures $1 \mathrm{D}-\mathrm{E})$. Control transthoracic echocardiography showed good valve function (peak and mean tricuspid gradient was 10 and $6 \mathrm{~mm} \mathrm{Hg}$ respectively). The patient showed significant clinical improvement and was discharged home 7 days later.
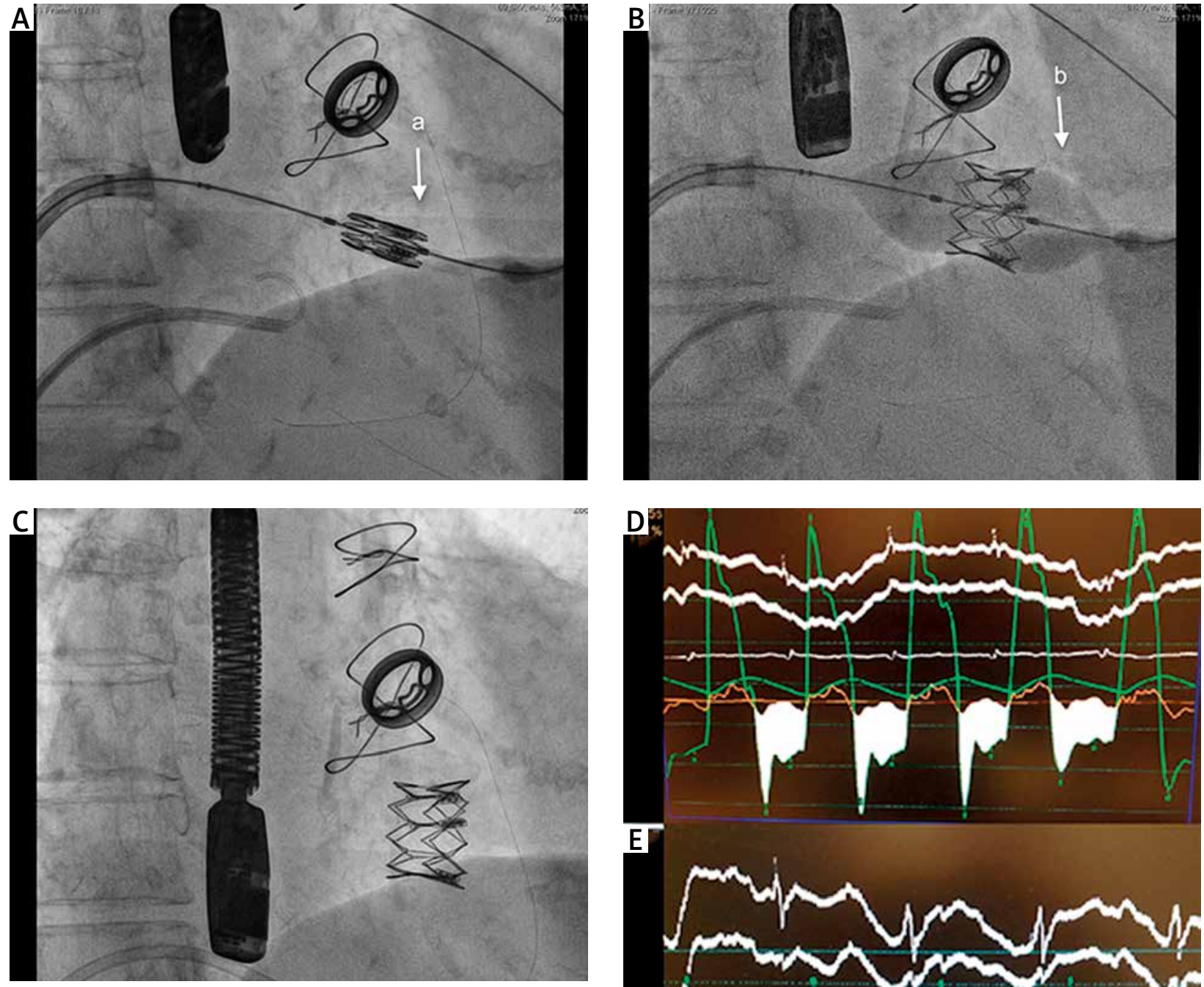

$\bar{E}$

Figure 1. Panels A, B and C illustrate the successive steps of the SAPIEN XT valve implantation. In detail: A - positioning of the SAPIEN XT valve (a) in the old tricuspid bioprosthesis, $\mathbf{B}$ - valve deployment during rapid pacing (b), $\mathbf{C}$ - the final result. Panels $\mathbf{D}$ and $\mathbf{E}$ demonstrate simultaneous right atrial pressure (yellow line) and right ventricular pressure (green line) before (D) and after (E) the SAPIEN XT implantation

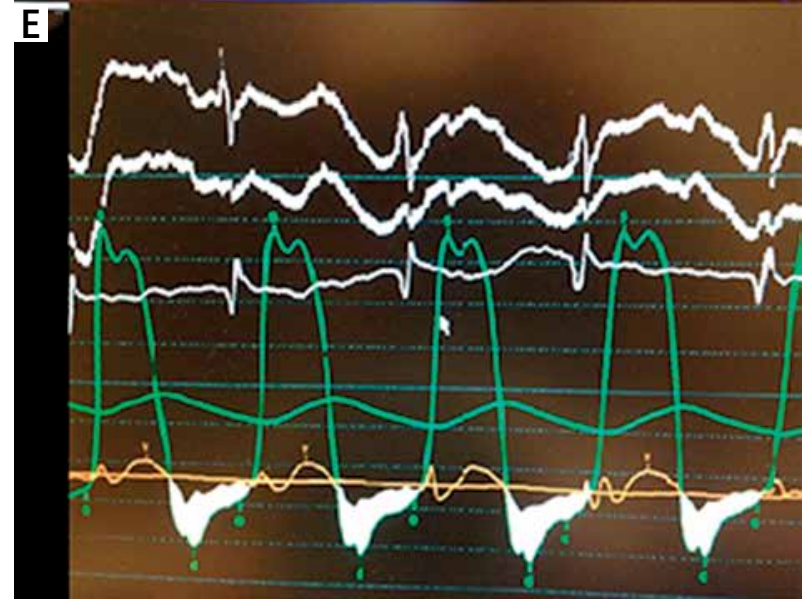



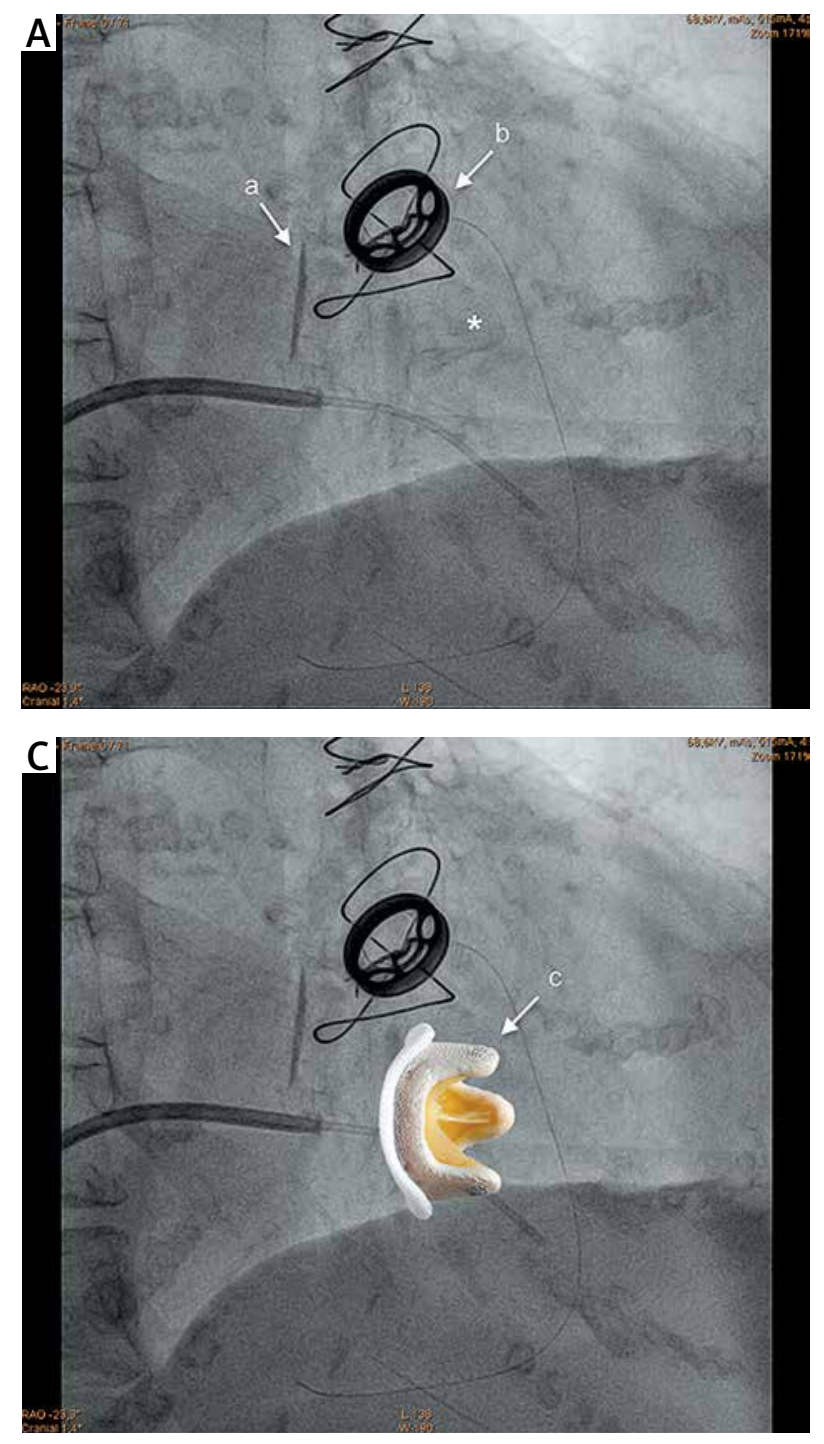

\section{Discussion}

In our case rapid ventricular pacing was indispensable, because the position of the system was unstable in the tricuspid ring in relation to heart movement. Neither transvenous right ventricular nor transarterial left ventricular pacing was applicable. Transcoronary sinus pacing could be a possible option [5], but this would require extra-procedural time, additional venous puncture and additional risk of failure to accomplish successful implantation $[6,7]$. We found the noninvasive, external pacing as a very useful option in an anesthetized patient. The pacing sticker-pads are routinely placed for such procedures in our cath lab. To our best knowledge this was the first reported noninvasive rapid pacing used for balloon expandable valve implantation. The original Medtronic Intact Valve bioprosthesis is supposed to be completely radiolucent. Contrary to the report of Calvert et al. [5], our 20-year-old TBP was perfectly seen on cine, due to valve tissue calcifications (Figure 2 A). The SAPIEN

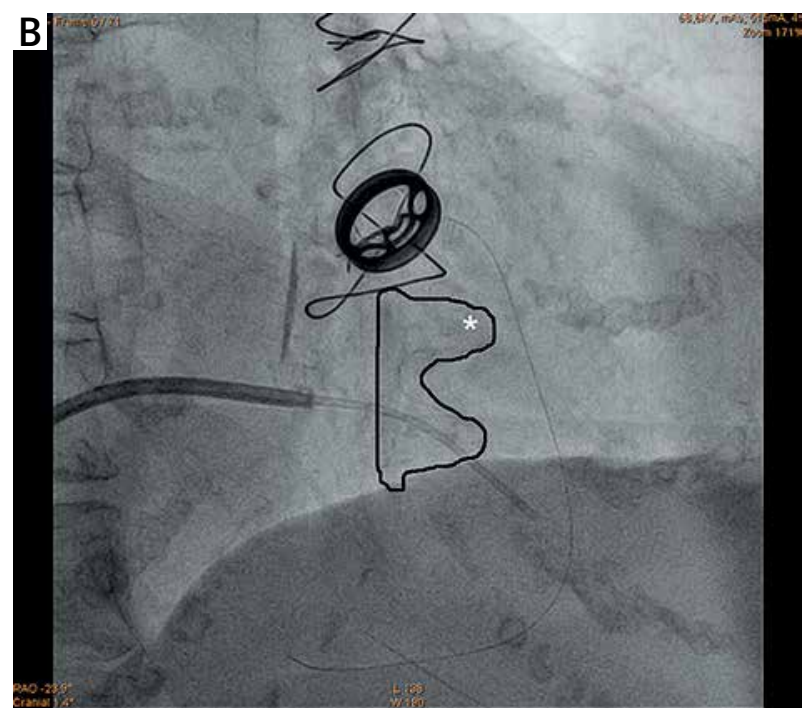

Figure 2. Panels A, B and C show the same fluoroscopy image recorded before the Edwards SAPIEN XT implantation. A - The old tricuspid bioprosthesis with visible calcifications $\left(^{*}\right)$, mitral (a) and aortic (b) prostheses. B - The bioprosthesis contour $\left.{ }^{(}\right)$. C - The bioprosthesis picture (c) overlaid on the fluoroscopic image

$\mathrm{XT}$ could be precisely positioned within the TBP relying on the fluoroscopy images, with TEE used to confirm SAPIEN XT position and function rather than to guide the procedure (Figure 3).

\section{Conclusions}

This case report describes a transfemoral Edwards SAPIEN XT valve implantation in a degenerated tricuspid bioprosthesis in a high-risk surgical patient resulting in gradient reduction and relief of symptoms. Technical challenges associated with invasive pacing were solved by non-invasive external pacing. Surface electrodes are commonly used clinically, in applications such as defibrillation or cardiac pacing. Occasionally they can cause pain, tissue damage, rash, or burns on the skin. In our case transcutaneous pacing was the method of choice because of difficulty placing a wire through the degenerated tricuspid valve prosthesis. General anesthesia provided pain relief during pacing. To avoid skin damage 

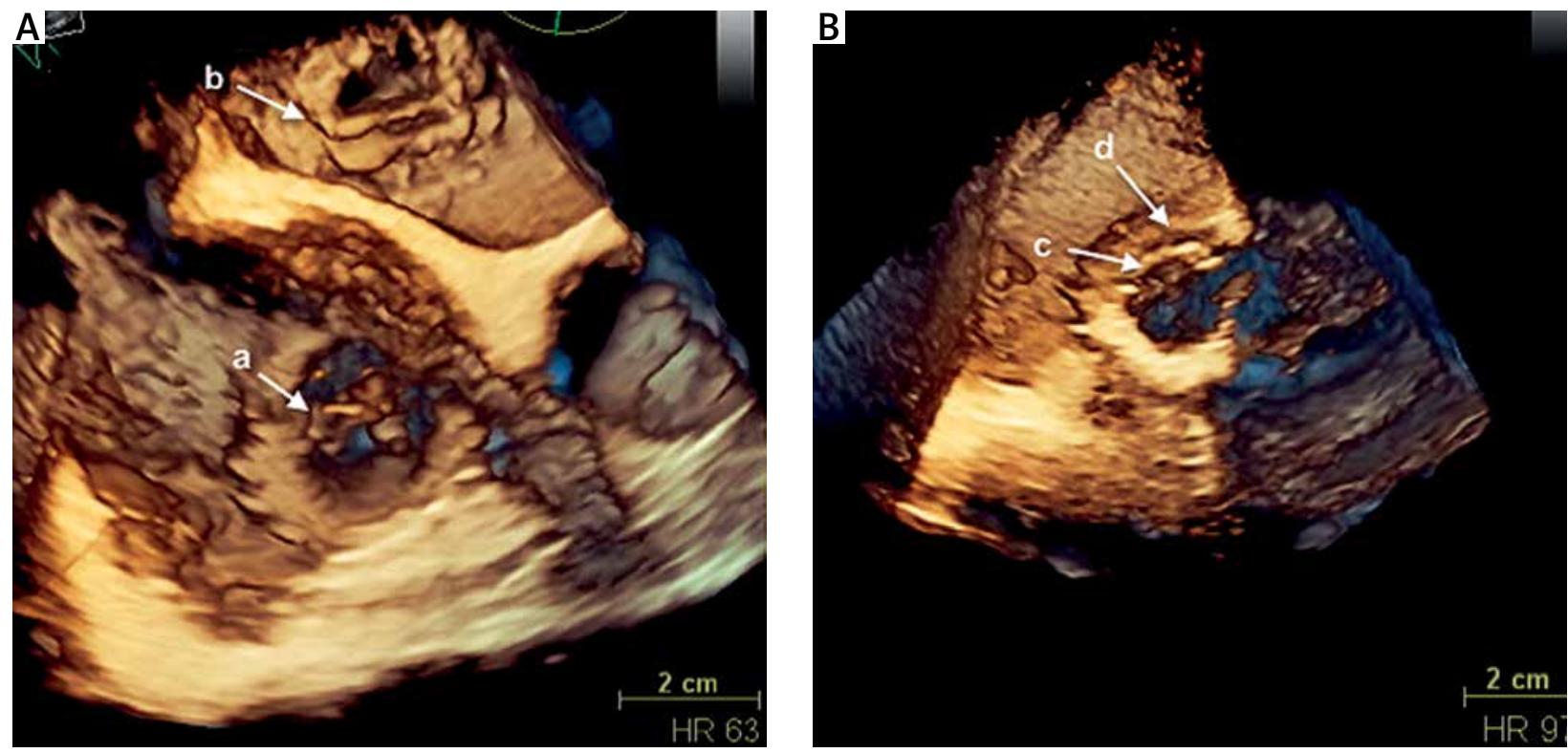

Figure 3. A - 3D TEE view before treatment demonstrates stenotic bioprosthetic valve in tricuspid position with degenerated leaflets (a) and prosthetic mitral valve with the leaflets opened (b). B - 3D TEE view of the SAPIEN XT valve (c) deployed inside the old tricuspid bioprosthesis (d)

underneath the electrodes it is necessary to evaluate the skin after the first $30 \mathrm{~min}$ and periodically. As energy density is low, the maximum temperature is kept within safe limits and a safe current distribution is provided [8]. In our case the overall pacing time was only several minutes and there were no signs of skin injury. In high-risk patients, such as those with low left ventricular ejection fraction and severe coronary artery disease, adenosine injection could represent an alternative to rapid ventricular pacing. In these patients, rapid pacing may induce myocardial ischemia or cause ventricular arrhythmias. However, adenosine is less predictable, short acting and is not recommended as a safe and effective method of heart stabilization during valve implantation. The time needed for transcutaneous tricuspid valve implantation is longer than the time of asystole provided by adenosine injection. Moreover, frequent occurrence of ventricular ectopic contractions during valvuloplasty may result in valve displacement. Prolonged AV block requiring transient pacing or failure to achieve asystole can also occur [9].

In summary, in the future, noninvasive transcutaneous pacing might be routinely used, saving time and reducing complications.

\section{Conflict of interest}

The authors declare no conflict of interest.

\section{References}

1. Hon JKF, Cheung A, Ye J, et al. Transatrial transcatheter tricuspid valve-in-valve implantation of balloon expandable bioprosthesis. Ann Thorac Surg 2010; 90: 1696-7.
2. Godart F, Baruteau AE, Petit J, et al. Transcatheter tricuspid valve implantation: a multicentre French study. Arch Cardiovasc Dis 2014; 107: 583-91.

3. Ribichini F, Pesarini G, Feola M, et al. Transcatheter tricuspid valve implantation by femoral approach in trivalvular heart disease. Am J Cardiol 2013; 112: 1051-3.

4. Mortazavi A, Reul RM, Cannizzaro L, Dougherty KG. Transvenous transcatheter valve-in-valve implantation after bioprosthetic tricuspid valve failure. Tex Heart Inst J 2014; 41: 507-10.

5. Calvert PA, Himbert D, Brochet E, et al. Transfemoral implantation of an Edwards SAPIEN valve in a tricuspid bioprosthesis without fluoroscopic landmarks. Eurointervention 2012; 7: 1336-9.

6. Yoda M, Hansky B, Koerfer R, Minami K. Coronary sinus dissection during left ventricular pacing electrode implantation. Ann Thorac Cardiovasc Surg 2007; 13: 275-7.

7. Swampillai J, Heald SC, Devlin GP, Stiles MK. Ventricular pacing via the coronary sinus in a patient with a mechanical tricuspid valve prosthesis. J Arrhythm 2011; 27: 343-6.

8. Besio W, Sharma V, Spaulding J. The effects of concentric ring electrode electrical stimulation on rat skin. Ann Biomed Eng 2010; 38: 1111-8

9. Davidavicius G, Chieffo A, Shannon J, et al. A high dose of adenosine to induce transient asystole for valvuloplasty in patients undergoing transcatheter aortic valve implantation (TAVI): is it a valid alternative to rapid pacing? A prospective pilot study. J Inv Cardiol 2011; 23: 467-71. 